\title{
LA IMPORTANCIA DE LA ADECUADA FORMACIÓN DEL TÉCNICO SUPERIOR EN EDUCACIÓN INFANTIL PARA UNA EDUCACIÓN RURAL DE CALIDAD, EXCELENCIA Y EQUIDAD
}

\author{
THE IMPORTANCE OF PROPER TEACHING OF THE TÉCNICO \\ SUPERIOR EN EDUCACIÓN INFANTIL FOR A RURAL EDUCATION \\ OF QUALITY, EXCELLENCE AND FAIRNESS
}

Ana María Moreno Aparicio ${ }^{a^{*}}$

Fechas de recepción y aceptación: 9 de octubre de 2019, 14 de abril de 2020

DOI: 10.46583/edetania_2020.57.502

Resumen: Los educadores infantiles en España tienen carencias en relación con su praxis en contextos rurales. Todo ello referido, principalmente, a la notoria falta de formación en los planes de estudio de Técnico Superior en Educación Infantil y a las carencias legislativas que no hacen alusión a una adecuada formación referente a la docencia rural.

Las disímiles condiciones del contexto en el que se hallan insertados estos centros escolares, la pujanza del maestro familiarizado con dicho medio y la investigación que tuvo como objetivo principal evidenciar la notoria falta de formación docente en relación con la educación rural han dispuesto que en el presente documento se pretenda dar a conocer, en su globalidad, el recorrido de la escuela rural en España, así como la adecuada formación de los futuros TSEI en el desempeño de técnicas e instrumentos de los procesos de aprendizajeenseñanza, con el objetivo principal de alcanzar dentro de un aula multinivel de primer ciclo de Educación Infantil rural la calidad, excelencia y equidad que verdaderamente la escuela rural se merece.

Palabras clave: TSEI, educación rural, formación docente, escuela rural.

\footnotetext{
${ }^{a}$ Facultad de Magisterio y Ciencias de la Educación. Universidad Católica de Valencia San Vicente Mártir.

* Correspondencia: Universidad Católica de Valencia San Vicente Mártir. Facultad de Magisterio y Ciencias de la Educación. Calle Sagrado Corazón, 5. 46110 (Godella), Valencia. España.

E-mail:amoa@mail.ucv.com
} 
Abstract: Preschool teachers in Spain have deficiencies when it comes to education in rural contexts. All of this is mainly due to the notorious lack of training in the Higher Technician in Early Childhood Education curriculum and to the legislative education deficiencies that do not allude, under any circumstances, to an adequate training at teaching in rural contexts.

The different conditions of the context in which these schools have been set up, the teacher's familiarity with this medium, and the research addressing the notorious lack of teacher training in rural education have triggered the makings of this paper, which generally tries to deal with the history of rural schools in Spain as well as teachers' proper training in this context, paying special attention to the TSEI's performance of techniques and instruments in the learning-teaching processes with the main aim of reaching a multilevel classroom of the first cycle of rural preschool education as well as the quality, excellence and fairness that the rural school truly deserves.

Keywords: TSEI, rural education, teaching educators, rural school.

\section{INTRODUCCIÓN}

La educación rural recorrió en España un largo trayecto educacional influenciado principalmente por los diversos cambios políticos que se dieron a lo largo de las diferentes décadas. A continuación, realizaremos un breve recorrido histórico por todas aquellas leyes educativas que influenciaron el desarrollo de la educación rural.

\subsection{La Ley Moyano}

Claudio Moyano fue el impulsor de la Ley Moyano. Esta ley fue promulgada durante el reinado de Isabel II en el año 1857, dispuesta por 307 artículos con 7 disposiciones transitorias; fue derogada el 4 de agosto de 1970 (Sevilla, 2007). "Teniendo una vigencia superior a los 110 años y desarrollándose por numerosos reglamentos en el transcurso de los años" (Berengeras y Vera, 2015, p. 3), la Ley Moyano consiguió crear una nueva concepción de la educación que cambiaría la manera de ver y comprender el mundo educativo en España, pues instauró lo que hoy en día conocemos como los principios de enseñanza gratuita.

Es en dicha ley donde, por un lado, nació la preocupación por la especialización docente $\mathrm{y}$, por otro lado, y en relación con la construcción de 
escuelas rurales, emergieron las llamadas escuelas unitarias (Sevilla, 2007). En la escuela unitaria de Moyano los discentes estaban agrupados por niveles distintos en una misma aula y bajo la dirección de un solo docente. En este periodo histórico-educativo, el alumnado era segregado por género, es decir, niños separados de niñas, pero en el caso de las unitarias eran aulas mixtas por la insuficiencia de discentes matriculados (Santamaría, 2012). Observamos que apenas hubo alusión alguna a esta tipología de escuelas, meramente fue mencionada en el artículo 102 de dicha ley una imperceptible preocupación e interés por la escuela rural del momento, lo que creó lo que hoy se conoce como escuela unitaria.

\subsection{La Educación rural en la II República}

El 14 de abril de 1931 se proclamó lo que se conoce como II República española, un movimiento político marcado por la pretensión de libertad y progresismo que pretendía abolir la larga trayectoria involucionista detonante de gobiernos anteriores. Este movimiento político duró apenas cinco años y finalizó por un golpe de Estado en el año 1936. Uno de los principales objetivos de la II República en relación con el sistema educativo español fue dotar a los territorios rurales de cultura mediante un aumento de presupuesto para la formación y construcción de escuelas rurales (Gimeno, 2011). Aquí ya podemos observar el origen de una preocupación por el acercamiento y mantenimiento de la cultura en contextos rurales. Es en este periodo cuando se comienzan a crear las escuelas unitarias rurales, ya formadas en la Ley Moyano, pero ahora marcadas por una pretensión de mejora y calidad.

Tal y como afirma Pérez (2000), el principal problema con el que tropezó la II República respecto al ámbito rural fue, por un lado, la falta de maestros en las escuelas, lo que perjudicaba gravemente a las escuelas de los contextos rurales. Por otro lado, la falta de escuelas físicas acrecentaba un elevado porcentaje de discentes sin escolarización debido principalmente a factores de índoles socioeconómicas como por ejemplo aquellos pequeños pueblos que no poseían escuela. Fue de este modo cuando por primera vez la figura del maestro rural comenzó a concebirse y perfilarse, de manera que se percibía al maestro como redentor del analfabetismo e incultura dentro de la sociedad 
rural. Al margen de todo ello, y a pesar de que se intentaba luchar contra la falta de escuelas en las poblaciones pequeñas, la precariedad escolar seguía latente. De ahí surgieron las llamadas Misiones Pedagógicas, promulgadas por la Institución Libre de Enseñanza (ILE) que Giner de los Ríos, junto con otros pedagogos, constituyó. Los principios de la ILE se basaron en los preceptos de ilustres pedagogos como Froebel, Pestalozzi o Montessori, que fueron los que influenciaron la elaboración del programa didáctico de las MM. PP. (Fernández, 2017). El objetivo primordial que estas misiones pretendían alcanzar, según el Decreto de 29 de mayo de 1931, era la difusión de la cultura general, la educación moderna de los maestros y la educación ciudadana de todas las pequeñas poblaciones, aldeas y villas. Además, cabe destacar la presencia de personas cultas como maestros, bibliotecarios, médicos, abogados y poetas y artistas, como Luis Cernuda, Alejandro Casona o Miguel Hernández, entre otros, que participaron de forma altruista para brindar la cultura al pueblo rural. Las Misiones Pedagógicas fueron un punto álgido en la cultura y folclore popular hasta la llegada del franquismo (Gimeno, 2011).

\subsection{Ley de Reforma de la Enseñanza Media de 1938}

Esta ley tuvo una durabilidad de 15 años y su pretensión era abolir todo aquello que la II República había instaurado en el territorio nacional (Berengueras y Vera, 2015). En referencia a la formación del maestro rural, no se alegó absolutamente nada para su mejoría. Lo único que se observó fue un ligero matiz en la reformulación de asignaturas de los educandos, mencionando que a la mujer se le instruiría en tareas del hogar como la sociedad rural requería, y al hombre en tareas más pragmáticas vinculadas también al campo (Lorenzo, 1998).

\subsection{Ley de Enseñanza Primaria 1945}

Es en esta ley cuando observamos un mayor interés por el modelo de educación rural, concretamente por la escuela unitaria. En el artículo número 21 de la Ley de Enseñanza Primaria quedan registrados aspectos como el dere- 
cho a la garantía de una escuela en cada pueblo, al menos en aquellos pueblos que tengan 250 habitantes censados.

Un aspecto muy importante que connotó una gran mejoría en el mundo rural, al pasarse de la segregación a la inclusión de los discentes, fue el nacimiento de las Escuelas Hogar. Eran centros educativos que amparaban en su infraestructura una especie de residencia-internado dirigido a todos aquellos discentes que a causa de sus condiciones geográficas y socioculturales no podían permitirse la asistencia a la educación, bien porque residían en aldeas rurales en las que los centros educativos estaban muy lejos de sus hogares, bien por motivos socioeconómicos, cuando las familias no podían resolver el transporte diario (Pérez, 2009).

\subsection{Ley de Formación Profesional Industrial de 1949}

Instaurada el 17 de julio de 1949, la Ley de Formación Profesional Industrial no tuvo ningún protagonismo en lo que concierne a la figura del maestro y a la escuela rural en sí, pero permitió visualizar de otra manera el contexto agropecuario propio del contexto rural. A través del Gobierno, con la creación de la Ley de Formación Profesional Industrial se dio la oportunidad de que las personas pudiesen especializarse en tareas agrícolas o ganaderas que propiciarían mejoras en las producciones y calidad de vida. Todo ello generó un tenue crecimiento demográfico en el censo de las poblaciones ya que muchas personas que estudiaban esta titulación se trasladaban a los pueblos para desempeñar sus funciones laborales.

\subsection{Ley General de Educación de 1970}

Tras todo lo relativo a lo educativo-rural en la etapa del franquismo, 19391975, se esperaba que la próxima reforma llevase consigo una mejora explícita de este contexto, pero no fue así. Santamaría (2012) sostiene que durante la LGE se pretendió la construcción de Colegios Nacionales, heterogéneo en edades y con elevado porcentaje de discentes. Es decir, todo lo contrario a la 
tipología de centros escolares rurales que coexistían en ese momento. Tras este concepto se ocultaba una axiomática razón socioeconómica: erradicar las pequeñas escuelas del mundo rural con la finalidad de reducir la demografía rural y alcanzar una mayor plétora laboral en áreas urbanas.

\subsection{Ley Orgánica 8/1985, de 3 de julio, del Derecho a la Educación}

Con la llegada de la LODE se instauró el Real Decreto de Educación Compensatoria, lo que aseguraría el derecho a la educación de todos los educandos, independientemente de sus condiciones socioeconómicas, geográficas, etc. Se trataba de un programa que contemplaba de manera positiva las desigualdades y privaciones que durante años atrás el sistema educativo había oprimido con sus respectivas legislaciones. Referente a la educación rural se propusieron diversos proyectos que fueron los precursores de lo que hoy en día concebimos como colegios rurales agrupados (CRA). Estos proyectos fueron los conocidos planes de apoyo a la escuela rural, proyectos experimentales para zonas rurales de la Comunidad Valenciana o planes de actuación para la escuela rural en Andalucía, que perseguían la finalidad de alcanzar la calidad, excelencia y equidad que la escuela rural se merecía.

\subsection{Ley Orgánica 1/1990, de 3 de octubre, de Ordenación General del} Sistema Educativo

La LOGSE se encargó de sustituir lo establecido por la LGE, regulaba la estructura y organización de los niveles no universitarios (Santamaría, 2012). Con relación al ámbito educativo rural, partía de una visión pesimista y aciaga en comparación con las áreas urbanas. Es por todo ello que desde la LOGSE se quiso garantizar la calidad e igualdad de oportunidades en el contexto rural y los discentes rurales fueron escolarizados en CRA. Es en dicha legislación donde apareció el concepto de crasificación masiva, es decir, la construcción masiva de CRA en territorio rural (Santamaría, 2014). 


\subsection{LOE, Ley Orgánica 2/2006, de 3 de mayo}

Esta ley presenta un planteamiento muy análogo a la LOGSE de 1990, en relación con la estructura, metodología, organización y atención a la diversidad en los centros escolares. Concerniente a la educación rural, una de sus principales prerrogativas es que vela por la garantía de oportunidades para todos los discentes y para los centros educativos. Así mismo en su artículo 82, referente a la igualdad de oportunidades en el mundo rural, se hace mención a la importancia de esta tipología de centros y a cómo la Administración debe preocuparse y ocuparse de estos para una mejora educativa.

\subsection{LOMCE, Ley Orgánica 8/2013, de 9 de diciembre, para la mejora de la calidad educativa}

Con la LOMCE se origina un cambio en todo el sistema educativo, influenciando también en lo que se refiere a la educación rural. La LOMCE se preocupa y ocupa de lo pertinente a la calidad, excelencia y equidad en todo el marco educativo nacional. Por todo ello, aunque no se haga alusión explícita a la educación rural, sí que hubo una influencia en ella tanto a favor como en contra. La LOMCE ha mantenido en vigor diez artículos, los cuales han perjudicado la situación educativa rural. Pero es cierto que ha habido treinta oportunidades de mejora respecto a la LOE, en referencia a la educación rural, que servirían de mejora si el auge político y social aunaran sus fuerzas para elaborar e implantar un Plan Nacional de Educación Rural.

\section{LA ESCUELA RURAL ESPAÑOLA, TIPOS DE ESCUELAS}

En la actualidad, no hay una definición exacta en lo que se refiere al concepto de escuela rural, pero algunos autores, como Cano (2003), Boix (2004), Abós (2007) y Villanueva (2012), que poseen una gran trayectoria en referencia a la educación rural, han aunado sus diversas opiniones para configurar una definición más concisa y exacta de lo que propiamente es la escuela rural. 
De esta forma, podemos decir que la escuela rural es el conjunto de centros educativos, tanto de Educación Infantil como de Educación Primaria, que se hallan inmersos en contextos meramente marcados por la ruralidad, y que a su vez condicionan el carácter de la praxis educativa tanto a nivel institucional del propio centro educativo como a nivel de la propia aula. Asimismo, cabe destacar que la escuela rural es una escuela heteróclita si la comparamos con las escuelas urbanas y periurbanas, pues no es solo distinta, sino que también es recóndita. Posee su propia identidad y un elevado porcentaje de potencial educativo. Y así lo podemos observar a través de la OCDE en Pisa 2015, con altas tasas de potencial educativo ignoradas por ser provenientes de alumnado rural. Por todo ello en la revista Spotlight 9. Country Roads se ha investigado sobre los niveles de calidad educativos del mundo rural. Todos ellos incentivados por las aulas multiniveles, la proximidad entre la escuela y la comunidad, baja ratio y, lo más importante, los maestros con experiencia y formación en dicho enclave contextual.

\subsection{Escuela comarcal completa y escuela comarcal incompleta}

Las escuelas comarcales completas se ubican en poblaciones rurales que poseen un elevado porcentaje de personas en edad escolar censadas y residentes en la población. Son colegios que reciben discentes procedentes de diversos pueblos, villas o aldeas que por motivos geográficamente contextuales no poseen escuelas en el lugar en que residen. Es por todo ello que ofrecen servicio de trasporte escolar con el principal objetivo de facilitar a las familias el transporte entre los pueblos y los centros escolares (Carmena y Regidor, 1985).

En referencias a las comarcales incompletas, son centros educativos cuyo número de aulas no abarca todos los niveles educativos para cada curso y ciclo, substancialmente por la falta de alumnado en las poblaciones colindantes. Estas aulas configuran, por su idiosincrasia, las denominadas aulas multinivel, es decir, aulas que abarcan una ratio muy baja de alumnado y con un alumnado además procedente de diversos cursos educativos (García, 2014). 


\subsection{Escuela unitaria}

Las escuelas unitarias o centros de difícil cobertura son los casos más excepcionales dentro de los tipos de centros que configuran la llamada escuela rural. Son centros que únicamente poseen una sola aula en la que una baja ratio de alumnos de distintos niveles educativos permanece en ellas, por lo que su estructura pedagógica se enclava principalmente en la heterogeneidad emanada de las múltiples edades cronológicas, de las competencias curriculares y de los niveles de organización. Todos estos factores se dictaminan bajo una organización administrativa adaptada a las demandas inherentes del medio en el que se halla inmersa. Las escuelas unitarias albergan el cuantioso esfuerzo del maestro, las familias y el pueblo con el objetivo primordial de mantener con vida la vida cultural y social de toda una población (García, 2014).

\subsection{CRA, Colegio Rural Agrupado}

Varios son los autores que han ahondado en la concepción, organización y praxis diaria de un Colegio Rural Agrupado, entre ellos destacan Ponce de León, Bravo y Torroba (2000), Martín (2006), Tapia y Castro (2014) y La Orden 10/1999, de 15 de mayo, de la Conselleria de Cultura, Educación y Ciencia, que coinciden en que un CRA es una agrupación de centros educativos que abarcan las etapas de Educación Infantil y Educación Primaria, situados en contextos rurales y que poseen diversos aularios ubicados en poblaciones colindantes aunque institucional y burocráticamente funcionan como si fuese un solo centro escolar.

\subsection{CRIE, Colegio Rural de Innovación Educativa}

Los CRIE hacen referencia a los Colegios Rurales de Innovación Educativa. Son centros escolares rurales que se han ido implantando en la sociedad rural hace relativamente pocos años, siendo Teruel pionero en su implantación. 
Estos centros han sido creados por la Administración y cuyo objetivo principal es promover la convivencia a través de proyectos socioeducativos entre alumnos de CRA de distintas comarcas o comunidades autónomas.

\section{BREVES CARACTERÍSTICAS DE LA ESCUELA RURAL}

\subsection{Multinivelariedad}

Debido a la casuística del contexto rural, las aulas de las escuelas rurales poseen un carácter meramente marcado por el término multinivelariedad, es decir, son centros escolares cuyas aulas son albergadas por alumnado de diferente edad cronológica, y en las cuales el docente debe ir impartiendo las materias y adaptándolas a los diversos ritmos, niveles y circunstancias que caracterizan su grupo clase (Quílez y Vázquez, 2012).

Cabe señalar que la propia idiosincrasia de estas aulas hace que el proceso de aprendizaje-enseñanza sea un proceso más real, es decir, hace que el transcurso del aprendizaje y de la relación entre personas se aproxime y equipare más a la realidad de la vida adulta, ya que los discentes conviven y trabajan con iguales de edades cronológicas heterogéneas (Martín, 2006). El aprendizaje que se da en este tipo de aulas es un aprendizaje impregnado por el contagio inevitable y permanente de ayuda al otro, de la cooperación. Los mayores ayudan a los pequeños y los pequeños aprenden de estos (Bustos, 2013).

\subsection{Baja ratio}

Otra de las características manifiestas de las escuelas rurales es la baja ratio de alumnos, tanto por aulas como en la totalidad de matriculados en el centro. Al ser una ratio tan reducida, el proceso de aprendizaje-enseñanza es muy condescendiente, la temporalización de las actividades que deben desarrollarse suele ser muy dúctil y por lo tanto dan cabida a un aprendizaje vivencial y significativo que al mismo tiempo se ve enriquecido con una relación docente-discente muy individualizada. 
Al mismo tiempo, a muchos docentes que nunca han sido formados en la práctica de la escuela rural, en pedagogías más individualistas y activas, les genera angustia y desasosiego programar y ejecutar procesos para la puesta en marcha del aprendizaje y enseñanza. Pero también encontramos aquellos docentes que o bien por su situación personal o bien por su estilo docente perciben y vivencian la baja ratio como un auge positivo para el proceso de aprendizaje-enseñanza, aunque suele ser un porcentaje muy reducido frente a los docentes que desvirtúan este tipo de escuelas (Villanueva, 2012).

\subsection{Arraigo cultural}

El arraigo cultural es un hecho que lleva instaurado desde los asentamientos de las primeras poblaciones. Este fenómeno se extrapola también a los procesos educacionales de los educandos rurales. Desde la escuela rural no solo se trabajan los contenidos curriculares que impone la Administración, sino que también se trabaja un arraigo a la cultura que, aunque puede parecer extraño por todas las incursiones culturales-urbanas, todavía pervive y por lo tanto debe de trabajarse adecuadamente en el área educativa con el objetivo ecuánime de conservar las raíces culturales. El arraigo cultural debe ser el eje vertebrador del proceso de aprendizaje-enseñanza en las escuelas rurales.

\section{Perfiles de los usuarios directos con la escuela RuRAl española}

\subsection{Discentes}

El perfil comportamental del niño rural en España suele ser el de un niño muy noble, social y disciplinado que, a gran diferencia de los niños urbanos, posee una plena conciencia de los quehaceres cotidianos de la vida rural que le rodean. Se compromete con las tareas del hogar, los oficios agropecuarios de sus familias, etc. Se trata de alumnado que se adapta con gran rapidez a los cambios, como ejemplifica su capacidad para combinar su vida rural con la agregada urbanización progresiva que se está acusando en las poblaciones 
con la llegada de internet, de la telefonía móvil, de los dispositivos electrónicos, etc. Todas y cada una de las pericias de la vida en el contexto rural ayudan en el aula multinivel. Los pequeños imitan a los compañeros mayores, al igual que hacen los niños con los adultos en el contexto urbano. Predomina un elevado porcentaje de trabajo cooperativo, por lo que todo ayuda a diseminar, si es que los hay porque no es lo habitual, los conflictos entre los propios compañeros del aula (Bustos, 2011).

\subsection{Familias}

Siguiendo a Bustos (2007) nos adentramos en el perfil de las familias de contextos rurales. Suelen ser familias que poseen descendencia pasada en la población y que perduran cohabitando en esta por el fortísimo arraigo cultural y estrechos lazos con el resto de los habitantes. Alguna tipología diferente que podemos encontrar a las habituales son aquellas familias que proceden del extranjero y que han llegado hasta estos rincones del territorio nacional debido a subvenciones propuestas por ayuntamientos para atraer a jóvenes y niños a la población y reducir así la despoblación que hoy en día se conoce como la España vaciada.

\subsection{Docentes}

Bustos (2009) y Mutuberria (2015) nos inician en el perfil del docente rural. Un perfil protagonizado por jóvenes sin experiencia, muchos de ellos recién salidos de una oposición, y con una deficiente, o nula, formación en la praxis educativa rural, marcada además, en un elevado porcentaje, por prejuicios negativos tanto hacia la vida rural como respecto a la educación. El patrón comportamental del docente novel en la escuela rural suele ser siempre el mismo. En primer lugar, hay unas fases inicial y media que se caracterizan por la ansiedad, la depresión y el miedo; más tarde entran en una fase de complacencia, felicidad y armonía por la labor que están llevando a cabo, no solo directamente con el alumnado sino en relación con la trascendencia de esta en sus familias y en la población en general. Es por todo ello que se deben extraer 
connotaciones positivas y sobre todo reales, para extrapolarlas a las formaciones iniciales de cualquier docente que se está instruyendo y, de esta manera, paliar y erradicar los tópicos negativos de la escuela rural en nuestro país.

Durston (2004) nos anuncia que no solo las carencias personales o laborales influyen, también lo hace el aislamiento geográfico en el que se encuentran. Todo ello provoca un aislamiento y una reticencia frente al intercambio de metodologías, asistencias a cursos formativos, etc. La falta de conexión entre la parte formativa y la praxis educativa hace que el profesorado que llega a estos centros educativos tenga los patrones comportamentales que hemos señalado con anterioridad. Los prejuicios de aquellos docentes que nunca han llegado a estar en una escuela rural alimentan a los nuevos docentes un miedo e incertidumbre constantes que les acarrea el fracaso, casi evidenciable si hablamos de la práctica docente en un aula multinivel.

\section{LA NECESIDAD DE UN EDUCADOR DIFERENTE PARA UN CONTEXTO DIFERENTE}

Abós $(2007,2011)$ defiende que la formación del docente debe estar ligada a las características y contextos actuales de nuestro país, destacando entre ellos el contexto rural. Apuesta por que la formación del docente rural sea una formación más exhausta y rigurosa en la que se asuma la diversidad del aula como un proceso enriquecedor, ya que asumir las desigualdades no quiere decir adaptarse a ellas, sino intentar mejorarlas y superarlas. Solo asumiendo que la escuela rural es una escuela distinta, se estará encauzando un correcto proceso de aprendizaje-enseñanza que podrá ser foco dinamizador de cultura para toda la comunidad y será de gran utilidad para que el alumnado se integre en pie de igualdad con las diferentes formas sociales. Por todo ello surge la necesidad de incluir en los planes de estudio del Técnico Superior en Educación Infantil elementos de carácter teórico y práctico en los que se promulgue la educación rural como un modelo de escuela completamente diferente.

Tras haber observado en primera persona, durante la práctica docente, una carencia en los planes de estudio de Técnico Superior en Educación Infantil (temarios ausentes en los módulos de dicha titulación, ausencia y conceptualización errónea de aquellos pocos docentes que se atreven a hablar de ello), 
se inició una investigación que abarcó dos ejes fundamentales que hay que tener en cuenta para defender la necesidad de un maestro diferente en un contexto diferente: investigación bibliográfica que otorgara una base científica a todo el proceso de investigación llevado a cabo y un análisis cuantitativo sobre los diferentes módulos que configuran el título del TSEI, sobre la realidad actual que vivencian los futuros TSEI en su formación académica y sobre aquellos docentes rurales en activo que habían recibido, o no, una formación rural en su formación como docente.

Respecto al análisis de los diversos módulos del TSEI a través del Real Decreto 1394/2007, de 29 de octubre, por el que se establece el título de Técnico Superior en Educación Infantil y se fijan las enseñanzas mínimas, se pudo observar que en el artículo 6 de dicho Real Decreto se encontraban implícitas las cualificaciones profesionales que el futuro TSEI debía adquirir al finalizar el Ciclo Formativo de Grado Superior. En ellas se observaba, aunque no explícita sino implícitamente, que una adecuada formación del TSEI exige saber contextualizar el proceso de aprendizaje-enseñanza a los discentes de 0 a 3 años, lo que a su vez demanda al futuro TSEI saber dominar los diferentes contextos educativos que se pueden dar, para así individualizar la enseñanza. Por lo tanto, debería trabajarse el contexto rural en nuestro país entre otros tipos de contextos que no sean los urbanos o periurbanos ordinarios, aspecto que no se contempla en absoluto bajo ninguna editorial, por lo que no se está cumpliendo dicho RD. Al mismo tiempo, es necesario tener en cuenta el artículo 9 de este, en el que se especifican los objetivos generales que debe alcanzar el futuro TSEI, y de nuevo no explícita sino implícitamente, se demanda la necesidad de saber analizar y conocer el contexto educativo para individualizar el proceso de aprendizaje-enseñanza. Por lo tanto, se evidencia la necesidad de introducir el contexto rural y su desempeño en técnicas e instrumentos de e/a en la educación rural para un TSEI. Al mismo tiempo, se pasaron cuestionarios abiertos a un grupo de discentes que cursaban primero y segundo de ciclos formativos de Grado Superior en Técnico Superior en Educación Infantil sobre la educación rural y su formación al respecto. Los datos obtenidos pueden resultar impactantes, pero el $100 \%$ de los alumnos encuestados dijeron no haber recibido formación en dicho contexto y ni tan siquiera supieron connotar definiciones exactas de ciertas características básicas de la escuela rural. Ello evidencia y afirma que el alumnado del TSEI no 
recibe una formación completa y adecuada en materia de formación en diversos contextos, tal y como aboga el Real Decreto 1394/2007, de 29 de octubre, por el que se establece el título de Técnico Superior en Educación Infantil y se fijan las enseñanzas mínimas.

Así mismo, también se pasaron y analizaron encuestas a docentes rurales en activo sobre si ellos habían sido formados durante su trayectoria facultativa en este contexto educativo y se obtuvieron datos alarmantes en los que claramente demandaban la necesidad de introducir, en un módulo del grado superior en Técnico Superior en Educación Infantil, un temario que abarcase todo lo relacionado con la educación rural. Incluyendo desde historia de la educación rural, pasando por legislación, hasta llegar a la configuración de técnicas e instrumentos de aprendizaje-enseñanza para aulas multinivel del primer ciclo de Educación Infantil.

Todo ello configura la evidencia de nuestra hipótesis. Es necesario formar a un docente diferente para un contexto diferente como el que viene a ser el contexto socioeducativo de la escuela rural. Se hace imprescindible que el docente rural comprenda las implicaciones y significados que conlleva su tarea educativa en dicho contexto. Por todo ello, los planes de estudios del TSEI necesitan incluir elementos de carácter teórico y práctico en los que se promulgue la educación rural como un modelo de escuela completamente diferente, en el que la atención a la diversidad, los ritmos evolutivos de aprendizaje, las aulas multinivel, el respeto hacia las personas y el medio que les rodea, la autonomía y madurez del alumno, y la vinculación con su territorio, hagan que la escuela rural requiera un cuerpo docente completamente preparado para hacer frente a todas esas demandas, con el objetivo primordial de desarrollar en los discentes todas las competencias para un correcto proceso de enseñanza-aprendizaje, y de esta manera otorgar a la educación rural la calidad, excelencia y equidad que verdaderamente se merece.

\section{Conclusiones}

El presente trabajo está sustentado por el máster en Formación del Profesorado de Secundaria, Bachillerato, Formación Profesional y Enseñanza de Idiomas de la especialidad Intervención Socio-Comunitaria. La temática 
llevada a cabo a través de esta pequeña investigación sobre cómo formar a futuros técnicos superiores en Educación Infantil para otorgar a la educación rural la calidad, excelencia y equidad que merece ha sido todo un reto a la hora de recopilar información, de manera activa, en la práctica docente de un aula real de TSEI y en la elaboración de un análisis exhaustivo de todas aquellas leyes educativas que han influido de manera positiva o negativa en la educación rural y que, por lo tanto, han influenciado en los planes de estudio que integran la formación de la titulación de TSEI en nuestro territorio nacional.

La ausencia de conceptualización y la conceptualización errónea de la escuela rural en los discentes de técnico superior en Educación Infantil evidencian palmariamente que la formación dentro del título de Técnico Superior en Educación infantil no está siendo una formación íntegra y equitativa con las realidades contextuales latentes en nuestro país, aspecto que se ha podido corroborar en las diversas leyes por las que nuestro sistema educativo ha pasado. Es por ello que -según el Real Decreto 1394/2007, de 29 de octubre, por el que se establece el título de Técnico Superior en Educación Infantil y se fijan sus enseñanzas mínimas, y la Orden de 29 de julio de 2009, de la Conselleria de Educación, por la que se establece para la Comunitat Valenciana el currículo del ciclo formativo del grado superior en Técnico Superior en Educación Infantil-, hay que considerarlas ya reflejadas en el artículo 9 y en el anexo 1, respectivamente.

Del mismo modo, se ha podido observar y evidenciar que esos conocimientos previos que el alumnado de TSEI posee en relación con la educación rural, muy difusos y erróneos, no están siendo susceptibles de un cambio ni por parte del Ministerio de Educación, cuando elaboran los reales decretos o las órdenes para realizar los planes de estudio, ni por parte del profesorado de Formación Profesional, cuando podrían llevar a cabo una ampliación del temario. Los docentes de Formación Profesional no se muestran concienciados de esta realidad, y todo ello se debe al mismo motivo: una ausencia de formación en los planes de estudio a nivel general en la formación docente y la notoria desvinculación del contexto rural en todos los ámbitos, incluso el ámbito socioeducativo, desfavoreciendo el artículo 27, de libertad de enseñanza y derecho a la educación, de la Constitución española de 1978. Asimismo, las diversas legislaciones por las que la educación española ha transcurrido a lo largo de los años hasta la actualidad tampoco han contemplado, de una mane- 
ra ajustada al paradigma educativo rural, la realidad inmersa en las aulas de los centros escolares rurales, pretendiendo proponer en los diferentes cambios legislativos medidas paliativas, pero no resolutivas, para este tipo de contexto que existe pero que lamentablemente quizás no persistirá en nuestra realidad educativa, si seguimos contemplando la formación de docentes preparados para enfrentarse a contextos urbanos y periurbanos e ignorando la formación de docentes para la vida escolar rural.

A modo de síntesis final, queda demostrado que todavía queda un precipitoso y laborioso camino para que se abogue por una educación rural de calidad, excelencia y equidad. Para ello será esencial que las diversas legislaciones educativas que vayan concurriendo en los próximos años postulen un interés y un cuidado para que la formación de los futuros docentes contemple los diversos contextos y ámbitos de actuación y lograr así enfrentarse a un proceso de enseñanza-aprendizaje en el ámbito rural.

\section{BiBliografíA}

Aвós, O. (2007). La escuela rural y sus condiciones ¿tiene implicaciones en la formación del profesorado? Aula abierta 35(1y 2), 83-90.

Aвós, P. (2011). La escuela en el medio rural y su presencia en los planes de estudio de los grados de maestro en Educación Infantil y Primaria de las universidades españolas. Profesorado. Revista de currículum y formación del profesorado 15(2), 39-52.

Berengueras, M. y Vera, J. M. (2015). Las leyes de educación en España en los últimos doscientos años. Supervisión 21, 38, 3.

Borx, R. (2004). La escuela rural: funcionamiento y necesidades. Madrid: Praxis.

Bustos, A. (2007). Enseñar en la escuela rural aprendiendo a hacerlo. Evolución de la identidad profesional en las aulas multigrado. Profesorado. Revista de currículum y formación del profesorado 11(3), 1-26.

Bustos, A. (2009). Valoraciones del profesorado de escuela rural sobre el entorno presente. Revista Iberoamericana de Educación 48(6), 1-11.

Bustos, A. (2012). La escuela rural. Barcelona: Magina. 
Bustos, A. (2013). El espacio y el tiempo en la escuela rural: algunas consideraciones sobre el aula multigrado. Revista de investigación en la Escuela $79,31-41$.

Carmena, G. y Regidor, J. (1985). La Escuela en el medio rural. Madrid: Servicio de publicaciones del MEC.

CAno, J. (2003). La realidad de las escuelas rurales. Educar en Castilla-La Mancha 3, 1-2.

Constitución Española, de 29 de diciembre de 1978 (BOE núm. 311, 29-121978, pp. 29313-29424).

Durston, J. (2004). La participación comunitaria en la gestión de la escuela rural. Centro Interamericano de Investigación y Documentación sobre Formación Profesional, 1-22.

Fernández, E. (2017). La Institución Libre de Enseñanza, las Misiones Pedagógicas y las Colonias Escolares: sus acciones para la renovación y sus dificultades durante su implantación. Revista Muesca 8, 53-72.

GARCíA, F. J. (2014). Escuela, medio rural y diversidad cultural en un contexto global: currículum, materiales didácticos y práctica docente de Conocimiento del Medio: situación, límites y posibilidades en centros onubenses, tesis doctoral, Huelva, España: Universidad de Huelva.

Gimeno, P. (2011). Las Misiones Pedagógicas de la II República española (1931-1939). Revista Ensayo 8, 176-177.

Ley de Reforma de Enseñanza Media 1938, de 23 de septiembre (BOE núm. 085, 23-09-1938, pp. 1385-1395).

Ley de Ordenación de la Universidad 1943, de 29 de julio (BOE núm. 212, 31-07-1943, pp. 7403-7431).

Ley de Enseñanza Primaria 1945, de 17 de julio (BOE núm. 199, 18-07-1945 pp. 385-416).

Ley de Formación Profesional Industrial 1949, de 17 de julio (BOE núm. 198, 17-07-1949, pp. 3163-3164).

Ley 14/1970, de 4 de agosto, General de Educación y Financiación de la Reforma Educativa (BOE núm. 187, 06-08-1970, pp. 12525-12546).

Ley Orgánica 8/1985, de 3 de julio, reguladora del Derecho a la Educación (BOE núm. 159, 04-07-1985, pp. 1985-12978).

Ley Orgánica 1/1990, de 3 de octubre, de Ordenación General del Sistema Educativo (BOE núm. 1238, 04-10-1990, pp.28297-28942). 
Ley Orgánica 2/2006, de 3 de mayo, de Educación (BOE núm. 106, 04-052006, pp. 2006-7899).

Ley Orgánica 8/2013, de 9 de diciembre, para la mejora de la calidad educativa (BOE núm. 295, 10-12-2013, pp. 2014-12886).

MARTín, N. (2006). La escuela en el medio rural. Guías para padres y madres 21, 1-32.

Mutuberria, A. (2015). La escuela rural: ventajas y desventajas. Revista Artista Digital 54, 2-4.

Orden de 10 de mayo de 1999, de la Conselleria de Cultura, Educación y Ciencia, por la que se adaptan las normas contenidas en el Reglamento Orgánico y Funcional de las Escuelas de Educación Infantil y de los Colegios de Educación Primaria a los Colegios Rurales Agrupados (CRAs) (DOGV núm. 3518, 16.06.1999).

Orden de 29 de julio de 2009, de la Conselleria de Educación, por la que se establece para la Comunitat Valenciana el currículo del ciclo formativo de Grado Superior correspondiente al título de Técnico Superior en Educación Infantil (DOGV núm. 6093, 02-09-2009, pp. 32862-32895).

Ponce de León, A., Bravo, E. y Torroba, T., (2000). Los colegios rurales agrupados, primer paso al mundo docente. Revista Contextos Educación 3, 315-347.

PÉrEz, F. (2009). Las Escuelas Hogar: de la segregación a la "inclusión” educativa del alumnado de población ultradiseminada. XV Coloquio de Historia de la Educación, 1-12.

Real Decreto 1394/2007, de 29 de octubre, por el que se establece el título de Técnico Superior en Educación Infantil y se fijan sus enseñanzas mínimas (BOE núm. 282, 24-11-2007, pp. 48140-48177).

SANTAMARÍA, R. (2014). La escuela rural en la LOMCE: oportunidades y amenazas. Revista Supervisión 21(33), 1-26.

TAPIA, L. y CASTRO, P. (2014). Experiencia educativa: educar desde un CRA. Tendencias pedagógicas 24, 415-428.

Villanueva, A. (2012). La escuela rural valenciana. Supervisión 21(26), 115. 
